\title{
DISLIPIDEMIA EM PACIENTES COM DIABETES TIPO 2
}

\section{Vilma Souza do Nascimento Barbosa}

Universidade Federal de Mato Grosso do Sul (UFMS), Brasil.

\section{Leuciney Santana Gomes}

Universidade Federal de Mato Grosso do Sul (UFMS), Brasil.

\section{Danielly Cristina Andrade Palma}

Doutoranda pelo Programa de Pós-graduação em Ciências Ambientais (UNEMAT). Docente Adjunto Nível I da Universidade Federal de Mato Grosso do Sul (UNEMAT), Brasil.

E-mail: danielly_palma@yahoo.com.br
RESUMO: O objetivo do estudo foi analisar a presença de dislipidemias em diabéticos tipo 2 atendidos pelas Unidades Básicas de Saúde do município de Coxim-MS. Estudo transversal observacional com abordagem quantitativa, composto por pacientes diabéticos tipo 2 cadastrados no programa Hiperdia, maiores de 18 anos, ambos os sexos, e que realizaram durante o ano de 2014 ao menos um exame de colesterol total, triglicerídeo e glicemia em jejum. Os resultados demonstram que $53,4 \%$ dos indivíduos tinham associações entre diabetes e dislipidemias reforçando que Diabetes Mellitus influi no metabolismo de lipídeos corroborando para aparecimento da dislipidemia e que o estilo de vida, sedentarismo e fatores sociodemográficos influem diretamente nesses resultados. Diante dos achados desse estudo vale ressaltar a importância em conhecer e planejar ações voltadas para promoção e prevenção desses agravos.

PALAVRAS-CHAVE: Dislipidemia; Hiperglicemia; Doenças cardiovasculares.

\section{DYSLIPIDEMIA IN PATIENTS WITH DIABETES T YPE 2}

ABSTRACT: The objective of this study was to analyse the presence of dyslipidemias in diabetics type 2 assisted by the Basic Health Units in the city of Coxim-MS. It is a cross-sectional and observational study with a quantitative approach, composed of diabetic patients type 2 registered in the Hiperdia Program, over 18 years old, both sexes, and that had during the year of 2014, at least, a total cholesterol, triglycerides and blood glucose examination after fasting. The results show that $53.4 \%$ of the individuals had associations between diabetes and dyslipidemias emphasizing that Diabetes Mellitus influences on the metabolism of lipids corroborating for the dyslipidemia appearance and that the life style, sedentarism and socio-demographic factors influence straightly on these results. In the face of this study discoveries it is worth pointing out the importance of knowing and planning actions turned to the promotion and prevention of those health problems.

KEY WORDS: Dyslipidemia; Hyperglycemia; Cardiovascular Diseases.

\section{INTRODUÇÃO}

O crescimento da população acarreta mudanças no perfil nutricional e consequentemente mudanças no perfil epidemiológico com predomínio de doenças crônicas não transmissíveis (DCNT) em detrimento das infecciosas. Nesse novo contexto, as doenças 
cardiovasculares (DCV) têm sido apontadas como importante desfecho crônico, pois são a principal causa de morte no mundo. Segundo a Organização PanAmericana de Saúde, as DCV representaram, em 2012, $31 \%$ de todas as mortes em nível global (OPAS, 2016).

São vários os fatores de risco relacionados às $\mathrm{DCV}$, os mais importantes são os fatores comportamentais, que podem se manifestar de diferentes formas nos indivíduos, sendo duas delas, a dislipidemia e o Diabetes Mellitus tipo 2 (OPAS, 2016).

As dislipidemias são anomalias dos lipídeos no sangue e podem tanto ocorrer por causas primárias, quando de origem genética, quanto por causas secundárias, decorrentes de estilo de vida inadequado (FALUDI et al., 2017). O Diabetes Mellitus (DM) tipo 2, ou diabetes não insulinodependente, é uma doença metabólica que se traduz pelo aumento do nível de açúcar no sangue. Desenvolve-se, geralmente, em virtude dos maus hábitos alimentares, sedentarismo e estresse da vida urbana (SBD, 2017).

De acordo com a International Diabetes Federations (2015), o número de portadores da doença, hoje no mundo, é de aproximadamente 415 milhões de adultos. Em 2016, no Brasil, 8,9\% dos brasileiros receberam o diagnóstico do diabetes (BRASIL, 2017).

$\mathrm{O}$ aparecimento de complicações vem associado a um desequilíbrio dos níveis de glicose e, o tempo de duração da doença junto a sua descompensação aumenta os riscos para adquirir problemas micro e macrovasculares. Sabe-se ainda que a interação da hiperglicemia com dislipidemias e outras patologias determinam o curso clínico para retinopatia, neuropatia e nefropatia (microvasculares) e cardiopatias (BRASIL, 2014).

A dislipidemia no diabético pode estar associada à síndrome metabólica e, neste caso, a soma de múltiplos fatores de risco exige uma abordagem terapêutica rigorosa pelo impacto deletério sobre a qualidade de vida e risco cardiovascular (ALMEIDA et al., 2007).

Assim, este estudo teve como objetivo analisar a presença de dislipidemias em portadores de Diabetes Mellitus tipo 2 atendidos pelas Unidades Básicas de Saúde do município de Coxim-MS, além de comparar os perfis lipídicos com a glicemia média dos indivíduos.

\section{METODOLOGIA}

Trata-se de um estudo transversal observacional com abordagem quantitativa.

O estudo foi realizado no município de Coxim, que se situa na borda setentrional da bacia do alto Paraguai. Com população estimada de 33.323 habitantes e área de 6.409,224 km², Coxim é o maior município e também o mais populoso da região Norte de Mato Grosso do Sul. (IBGE, 2017).

A amostra foi composta por pacientes diabéticos, tipo 2, cadastrados no programa Hiperdia, maiores de 18 anos, ambos os sexos, e que realizaram durante o ano de 2014 ao menos um exame de colesterol total, triglicerídeo e glicemia em jejum no laboratório municipal de Coxim.

Após um levantamento nas Unidades Básicas de Saúde (UBS), por meio do programa Hiperdia, para obter o nome dos pacientes diabéticos, tipo 2, cadastrados no município, foi realizada uma busca no programa SIC WIN ${ }^{\circledR}$ utilizado pelo laboratório municipal, onde foram realizados todos os exames da rede pública do município. Nesta busca foram coletados os resultados dos níveis de colesterol total, triglicerídeos e de glicemia em jejum dos pacientes anteriormente cadastrados.

Os exames de colesterol total, triglicerídeos e glicemia em jejum foram realizados utilizando o método enzimático colorimétrico (Trinder) e o equipamento utilizado foi o $\mathrm{BIO} 200$. Os exames foram realizados com pacientes em jejum de 12 a 14 horas.

A coleta de dados ocorreu entre os meses de setembro e outubro de 2015 e foram submetidos à análise estatística descritiva e teste de correlação de Pearson por meio do software Microsoft Office Excel ${ }^{\circledR}$.

Atendendo a resolução 466/12 do Conselho Nacional de Saúde, o mesmo (quem?) foi encaminhado ao Comitê de Ética e Pesquisa com Seres Humanos e foi aprovado com o parecer número 1.209.430 em setembro de 2015.

\section{RESULTADOS}

No período de coleta estavam cadastrados, no programa Hiperdia, 430 pacientes com DM2. Seguindo 
os critérios de inclusão, 91 (21,16\%) pacientes não

A amostra foi composta por $67,55 \%$ de mulheres atenderam aos critérios e foram excluídos da amostra, totalizando 339 pacientes $(78,84 \%)$ aptos em participarem e $32,45 \%$ de homens. A idade variou entre 18 a 98 anos, do estudo.

Tabela 1. Distribuição de casos de DM2 por idade e sexo no município de Coxim (MS), 2015

\begin{tabular}{cccc}
\hline Idade & Homens & Mulheres & Total \\
\hline 18 a 29 & 2 & 3 & 5 \\
30 a 39 & 4 & 5 & 9 \\
40 a 49 & 15 & 30 & 45 \\
50 a 59 & 18 & 69 & 87 \\
60 a 69 & 31 & 59 & 90 \\
$\geq 70$ & 40 & 63 & 103 \\
\hline Total & $\mathbf{1 1 0}$ & $\mathbf{2 2 9}$ & $\mathbf{3 3 9}$ \\
\hline
\end{tabular}

Dos 339 pacientes, 94,1\% apresentaram níveis de glicemia elevado, destes 40,7\% hiperglicemia sem dislipidemia e 53,4\% com hiperglicemia e dislipidemia associadas (Tabela 2).

Tabela 2. Níveis de glicemia em uma amostra de 339 pacientes com DM2 no município de Coxim-MS em 2015

\begin{tabular}{cccc}
\hline Níveis de glicemia & Homens (\%) & Mulheres (\%) & Total (\%) \\
\hline \multirow{2}{*}{ Normal } & 8 & 12 & 20( \\
& $(2,36 \%)$ & $(3,54 \%)$ & $5,9 \%)$ \\
Hiperglicemia & 41 & 97 & 138 \\
& $(12,09 \%)$ & $(28,61 \%)$ & $(40,7 \%)$ \\
Hiperglicemia + Dislipidemia & 61 & 120 & 181 \\
& $(18 \%)$ & $(35,4 \%)$ & $(53,4 \%)$ \\
\hline
\end{tabular}

Os resultados médios de glicemia, colesterol total e triglicerídeos encontrados podem ser observados na Tabela 3 .

Tabela 3. Valores médios e desvio padrão de glicemia, colesterol total e triglicerídeos de uma amostra de 339 pacientes diabéticos, no município de Coxim-MS, 2015

\begin{tabular}{ccc}
\hline Exame & Homens & Mulheres \\
\hline Glicemia $(\mathrm{mg} / \mathrm{dL})$ & $202( \pm 86)$ & $201( \pm 78)$ \\
Colesterol Total $(\mathrm{mg} / \mathrm{dL})$ & $225( \pm 72)$ & $224( \pm 65)$ \\
Triglicerídeos $(\mathrm{mg} / \mathrm{dL})$ & $248( \pm 160)$ & $219( \pm 111)$ \\
\hline
\end{tabular}

Outro resultado observado foi que não houve correlação entre os níveis de glicemia e os níveis de colesterol total e triglicerídeos em ambos os sexos (Figura 1).

Entre as mulheres para ambas as correlações o valor do coeficiente de correlação de Pearson foi $r=0,41 \mathrm{e}$ entre os homens a correlação entre glicemia e colesterol total foi $r=0,53$ e entre glicemia e triglicerídeos $r=0,43$. 
a

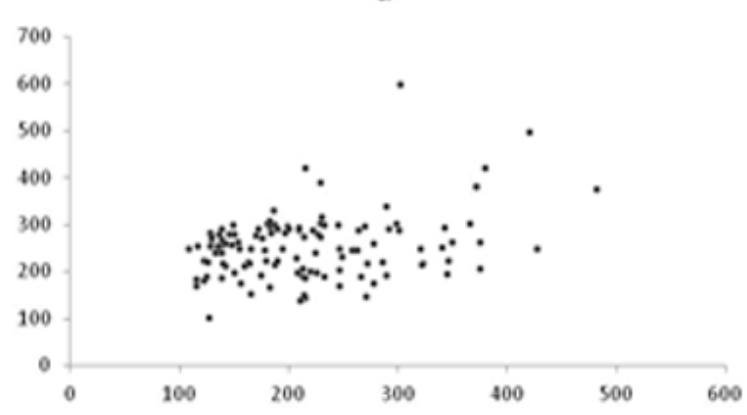

c

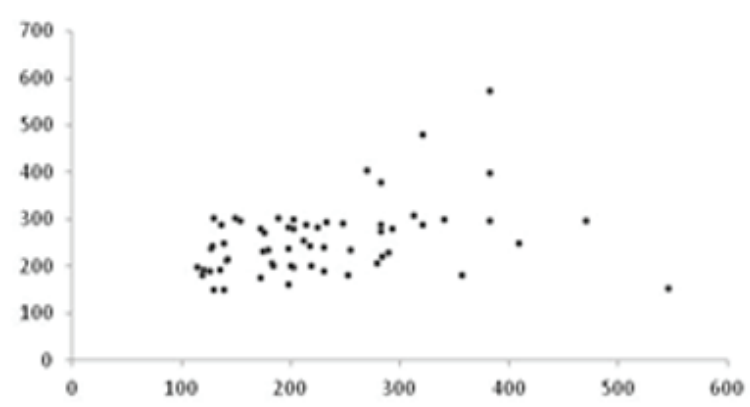

b

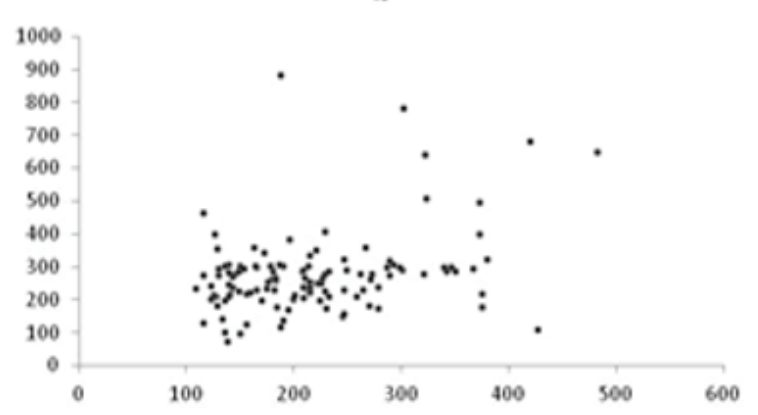

d

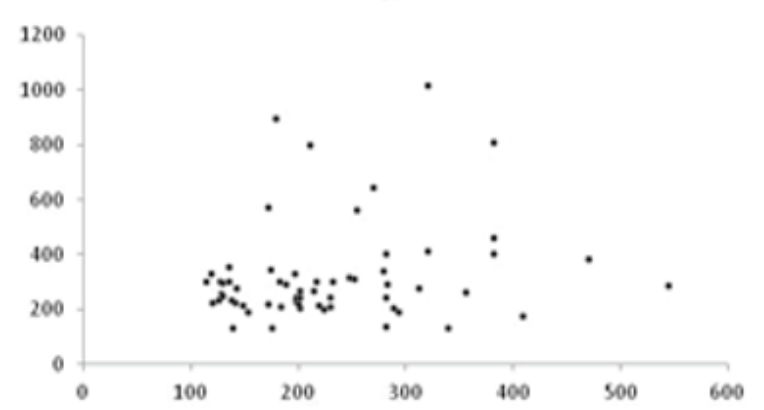

Figura 1. Diagramas de dispersão. a) correlação entre glicemia e colesterol total no sexo feminino; b) correlação entre glicemia e triglicerídeos no sexo feminino; c) correlação entre glicemia e colesterol total no sexo masculino; d) correlação entre glicemia e triglicerídeos no sexo masculino.

\section{DISCUSSÃO}

Os idosos apresentaram maior prevalência de DM2, totalizando $57 \%$ dos casos, como observado na Tabela 1. O aumento da resistência à insulina, pela redução da massa muscular, e a redução da função do pâncreas, é uma alteração decorrente do processo de envelhecimento que influencia a prevalência da doença nessa faixa etária (CDC, 2014).

O tipo mais prevalente de diabetes em idosos é o tipo 2 da doença, sendo mais raro com a idade o diagnóstico do tipo 1 que é associado a pessoas mais jovens. Relacionada ao portador de DM2, existe uma diferenciação arrolada ao seu IMC. Em pacientes idosos com IMC inadequados e obesos observa-se que há resistência à glicose mediada por insulina, e no caso dos idosos magros há alteração na liberação de insulina, e isso serve para moldar a construção do tratamento considerando a necessidade de cada indivíduo (VIEGAS, 2009).

A obesidade, como já se sabe, é um fator de risco para DM2 e com o envelhecimento ela se torna mais comum pela diminuição da prática de atividades físicas que são consequências desse processo de envelhecer, ou seja, a falta dessa atividade que diminui naturalmente e com o passar dos anos aumenta a possibilidade de DM2 como também de outros índices como os lipídeos (VIEGAS, 2009).

Outro fator importantíssimo é a presença de doenças ou comorbidades que juntas afetam em fatores ligados à liberação de insulina e metabolismo de glicose $\mathrm{e}$ resistência (VIEGAS, 2009).

Foi encontrado também DM2 em adultos jovens, $1,8 \%$ da amostra estão na faixa etária de 18 a 29 anos. Anteriormente, o diagnóstico nessa fase era caracterizado como DM1, porém cada vez mais vem sendo distribuída 
de forma parecida com a fase adulta que se mostra voltada ao DM2 (GABBAY; CESARINI; DIB, 2003), isso se dá pela similaridade de hábitos entre adultos, crianças e adolescentes. O sedentarismo e os maus hábitos alimentares têm gerado nessa faixa etária o aumento de obesos ou com sobrepeso que corrobora para a detecção do DM2 assim como em adultos com mais de 40 anos (CORRÊA, 2004).

A dislipidemia é uma alteração do metabolismo de lipoproteínas circulantes no sangue que no DM2 é causada pela resistência à insulina e à obesidade. Diabéticos com níveis glicêmicos descompensados têm maior probabilidade de desenvolver as dislipidemias, pois, a alta concentração de ácidos graxos livres em diabéticos é um fator que implica em redução da sensibilidade à insulina e aumenta a produção de gorduras (PEREIRA, 2011).

A hiperglicemia contribui para o desequilíbrio de lipídios circulantes, já que a resistência à insulina permite um metabolismo inadequado na produção desses lipídios, identifica-se que a condução correta a partir da regulação dos níveis de glicemia com comportamentos que incluem boa utilização de medicamentos hipoglicemiantes e exercícios físicos proferem melhora no aspecto de dislipidemia em DM2 (PINHO, 2015).

Carolino (2008) explica que o aparecimento desses números sugere que os pacientes não têm aderido a dietas adequadas, bem como não realizam regularmente exercícios físicos. Pereira (2011) reforça a associação da obesidade ao DM2 e dislipidemias, utilizando como solução a implementação de hábitos saudáveis.

As médias encontradas foram muito próximas para ambos os sexos, o que corrobora com Morais, Chieccio e Freitas (2013), que descrevem que os níveis de dislipidemias são elevados em ambos os sexos, principalmente após os 40 anos de idade, mas mostram também que há alterações nas frações lipídicas de acordo com fatores sociodemográficos, em que baixa escolaridade e renda contribuem diretamente para o excesso de peso que é um fator agravante para dislipidemias.

O mesmo estudo indica grande porcentagem de mulheres com a doença, o que também foi encontrado neste trabalho, porém os autores ressaltam que isso se evidencia no fato de a maior procura dos serviços de saúde ser pela população feminina, mas não que elas fossem as mais acometidas. Também expõe que existem fatores que têm atacado mais mulheres, como os baixos índices de HDL, levando a maiores riscos de problemas cardiovasculares. A Pesquisa Nacional de Saúde (PNS), em 2013, identificou uma prevalência de DM2 1,3 vezes maior em mulheres que em homens no Brasil.

Nos dados apresentados por Menezes et al. (2014), mulheres também têm maior índice de obesidade e, como visto na literatura, a obesidade está associada ao DM2 e níveis elevados de lipídeos, sendo assim as Diretrizes da Sociedade Brasileira de Diabetes (20152016) recomendam a redução do peso como principal medida não farmacológica para controle do DM e dislipidemias, porém há dificuldades na adesão a este tipo de tratamento não farmacológico.

Ainda no estudo de Menezes et al., (2014), a ingestão de bebidas alcoólicas por mulheres gera maior consequência em relação a patogênese diabética; os autores utilizam da literatura para interpretar a associação e relata a falta de exatidão dos mecanismos de ação do álcool sobre o diabetes, mas esclarece que há estudos que documentam forte ligação entre o consumo de álcool e liberação de adipocinas e marcadores de inflamação que estão ligados à doença.

A literatura aponta que a hiperglicemia influi na metabolização de lipídeos aumentando a lipólise e a quantidade de ácidos graxos livres (SCHOFIELD et al., 2016). Os resultados apresentados mostram correlação fraca entre glicemia, colesterol total e triglicerídeo. Esses resultados podem ser justificados pelo fato de os níveis glicêmicos terem sido avaliados em jejum e indicarem o índice momentâneo da glicose, o que não discrimina sua condição geral de doença. Quanto ao colesterol, não foram quantificadas as frações, podendo mostrar resultados que indicam que não exista correlação, pois os índices de lipídeos poderiam se mostrar menores enquanto os glicêmicos se mostrariam maiores ou viceversa. 


\section{CONCLUSÃO}

Dentre os pacientes atendidos pelo programa Hiperdia não houve diferença significativa nos valores de glicemia, colesterol total e triglicerídeo em ambos os sexos. Contudo, foi observado que mulheres predominaram no número de pacientes cadastrados no programa.

Observou-se que os valores médios de glicemia, colesterol total e triglicerídeo estavam elevados.

Maiores inferências poderiam ter sido feitas caso o laboratório municipal disponibilizasse exames com as frações lipídicas o que contribuiria para as análises realizadas. Assim, se fazem necessários outros estudos capazes de verificar outras variáveis que interferem no curso e na relação do Diabetes Mellitus com a dislipidemia.

\section{REFERÊNCIA}

ALMEIDA et al. Dislipidemias e Diabetes Mellitus: fisiopatologia e tratamento. Rev. Ciênc.Méd. v.16, n. 4-6, Campinas, 2007.

BRASIL. Ministério da Saúde. Secretaria de Vigilância em Saúde. Departamento de Vigilância de Doenças e Agravos não Transmissíveis e Promoção da Saúde. Vigitel Brasil 2016: vigilância de fatores de risco e proteção para doenças crônicas por inquérito telefônico: estimativas sobre frequência e distribuição sociodemográfica de fatores de risco e proteção para doenças crônicas nas capitais dos 26 estados brasileiros e no Distrito Federal em 2016. Brasília: Ministério da Saúde, 2017.

BRASIL. Ministério da Saúde. Secretaria de Atenção à Saúde. Departamento de Atenção Básica. Estratégias para o cuidado da pessoa com doença Brasília: Ministério da Saúde, 2014. 162p. (Cadernos de Atenção Básica, n. 35). Disponível em: < http://bvsms.saude.gov. br $>$. Acesso em: 18 out 2015.

CAROLINO et al. Fatores de risco em pacientes com Diabetes Mellitus tipo 2. Rev. Latino Am Enfermagem. v. 16, n. 2, Maio-Abril, 2008.

CDC - Centro de Diabetes Curitiba. 2014. Diabetes no paciente idoso. Disponível em: <http://www. centrodediabetescuritiba.com.br/artigos/diabetes-nopaciente-idoso/> . Acesso em: 25 nov. 2017.

CORRÊA H. FERMANDA. Diabetes Mellitus tipo 2 na criança e no adolescente. Adolescência \& Saúde, v.01, n.2, jun. 2004.

MILECH, Adolfo et al. Diretrizes da Sociedade Brasileira de Diabetes (2015-2016). São Paulo: A.C. Farmacêutica, 2016.

FALUD, A. A. et al. Atualização da diretriz brasileira de dislipidemias e prevenção da aterosclerose - 2017. Arquivos Brasileiros de Cardiologia, v. 109, n. 2 (supl. 1), 2017.

GABBAY, M., CESARINI, R. P.; DIB, A. S. Diabetes Mellitus do tipo 2 na infância e adolescência: revisão da literatura. Jornal de Pediatria, v. 79, n. 3, 2003.

IBGE. Instituto Brasileiro de Geografia e Estatística. Cidades. Disponível em:<https://cidades.ibge.gov.br/ brasil/ms/coxim/panorama > . Acesso em: 25 nov. 2017.

INTERNATIONAL DIABETES FEDERATION. Disponível em: < http://www.idf.org > . Acesso em: 5 mar. 2015.

MENEZES et al. Diabetes Mellitus e fatores associados em idosos residentes em Campina Grande, Paraíba. Rev. Bras. Geriatr. Gerontol., v. 17, n. 4, 2014.

MORAES A. S.; CHECCHIO V. M.; FREITAS M. C. I. Dislipidemia e fatores associados em adultos residentes em Ribeirão Preto, SP. Resultados do Projeto EPIDCV. Arq Bras Endocrinol Metab. v. 57, n. 9, 2013.

OPAS - Organização Pan-Americana de Saúde. Doenças cardiovasculares. 2016. Disponível em: <http://www. paho.org/bra/index.php?option $=$ com_content\&view $=$ art icle\&id $=5253$ : doencas-cardiovasculares\&Itemid $=839>$. Acesso em: 27 nov. 2017.

PEREIRA, R. A relação entre dislipidemias e Diabetes Mellitus tipo 2. Cadernos UniFOA, v. 17, dez. 2011. 
PINHO, L. et al. Hipertensão e dislipidemias em pacientes Diabetes Mellitus tipo2: uma revisão integrativa. Renome: Revista Norte Mineira de Enfermagem. v. 4, n. 1, 2015.

PNS - Pesquisa Nacional de Saúde 2013. Percepção do estado de saúde, estilo de vida e doenças crônicas: Brasil, Grandes regiões e unidades da federação. Disponível em: <https://biblioteca.ibge.gov.br/ visualizacao/livros/liv91110.pdf $>$. Acesso em: 25 nov. 2017.

SBD - Sociedade Brasileira de diabetes. Diabetes tipo 2. Disponível em: < http://www.diabetes.org.br/publico/ diabetes-tipo-2>. Acesso em: 25 nov. 2017.

SCHOFIELD, J. D. et al. Diabetes Dyslipidemia. Diabetes Ther. v. 7, p. 203-219, 2016.

VIEGAS, K. Prevalência de Diabete Mellitus na população de idosos de Porto Alegre e suas características sociodemográficas e de saúde. 2009. 197f. Tese (Doutorado em Gerontologia Biomédica) Pontifícia Universidade Católica do Rio Grande do Sul, Porto Alegre, 2009.

Recebido em: 2017-06-15

Aceito em: 2017-12-01 